\title{
A Persistent Foramen of Huschke: A Small Road to Misery in Necrotizing External Otitis
}

\author{
(D)W.L. van der Meer, (D) M. van Tilburg, (D) Mitea, and (D)A.A. Postma
}

\begin{abstract}
BACKGROUND AND PURPOSE: Necrotizing external otitis is a serious complication of external otitis with different spreading patterns. A persistent foramen of Huschke is a dehiscence located antero-inferior in the osseous external ear canal and posterior-medial to the temporomandibular joint. This dehiscence can facilitate extension of infection in an anterior pattern next to classic spread along the fissures of Santorini. The aim of this study was to define the prevalence and size of a persistent foramen of Huschke in patients with necrotizing external otitis.
\end{abstract}

MATERIALS AND METHODS: We retrospectively examined 78 CT temporal bone studies (39 patients with necrotizing external otitis, 39 control subjects). The side and presence of the foramen were noted, and its prevalence was calculated. The maximal width of the foramen of Huschke was measured in the axial plane and classified as subtle, mild, moderate, or extensive.

RESULTS: A persistent foramen of Huschke was present in 21 patients ( 26 ears) and 7 control subjects ( 9 ears). Prevalence was 50\% (20/40) and $11.5 \%(9 / 78)$ in affected ears of patients with necrotizing external otitis and control subjects, respectively. Almost all affected ears showed an anterior distribution pattern of necrotizing external otitis. The extensive dehiscence was most common in affected ears.

CONCLUSIONS: An anterior necrotizing external otitis spreading pattern is associated with the presence and increased size of a persistent foramen of Huschke. These findings facilitate the theory that a persistent foramen of Huschke is an additional risk factor the development of necrotizing external otitis.

ABBREVIATIONS: EAC $=$ external auditory canal; NEO $=$ necrotizing external otitis; $\mathrm{TMJ}=$ temporomandibular joint

$\mathbf{N}$ ecrotizing external otitis (NEO) is a rare-but-serious complication when a persistent external otitis, most commonly caused by the bacteria Pseudomonas aeruginosa ${ }^{1}$, fails to resolve. The infection can spread via fascial planes to surrounding soft tissues and bone structures. In severe cases, the infection affects intracranial structures and cranial nerves with persistent impairment after treatment. ${ }^{2}$ Patients at risk for NEO most often are elderly with diabetes and or are immunocompromised, and NEO can have a mortality rate up to $15 \% .{ }^{3}$ Hence, early recognition and tailored treatment with intravenous antibiotics could have a substantial impact on morbidity and mortality.

Received February 20, 2019; accepted after revision June 30.

From the Departments of Radiology and Nuclear Medicine (W.L.v.d.M., C.M., A.A.P.) and Otorhinolaryngology and Head and Neck Surgery (M.v.T.), Maastricht University Medical Center, Maastricht, the Netherlands; and School for Mental Health and Neuroscience (A.A.P.), Maastricht University, Maastricht, the Netherlands.

Please address correspondence to W.L. van der Meer, MD, Department of Radiology and Nuclear Medicine, Maastricht University Medical Center, P. Debyelaan 25, 6229 HX Maastricht, the Netherlands; e-mail: lieke.vander.meer@mumc.nl

http://dx.doi.org/10.3174/ajnr.A6161
NEO can be categorized according to an anterior, posterior, medial, or an intracranial spreading pattern or a combination of these aforementioned categories. ${ }^{4,5}$ The classification depends on the affected soft-tissue compartments and bone structures. ${ }^{5}$ In an anterior spreading pattern, the temporomandibular joint (TMJ), masticator space, parotid gland, or surrounding fat planes are involved (Figs 1 and 2). The posterior spreading pattern affects the mastoid process, and the medial spreading pattern affects the sphenoid, clivus, nasopharyngeal or pharyngeal muscles/fat, and cranial nerves IX, X, and XI. The intracranial pattern shows involvement of the jugular fossa, jugular vein, sigmoid sinus, and dura.

The spread of NEO from the external ear canal to its surrounding structures is thought to occur via the fissures of Santorini, also known as the incisurae cartilaginis meatus acustici, by extending through these osteocartilaginous or cartilaginous junctions of the external ear canal, spreading through fascial planes and blood vessels to surrounding compartments (Fig 3). Thus, early changes in NEO can be subtle and therefore easily overlooked. One of the early subtle findings is infiltration of the 


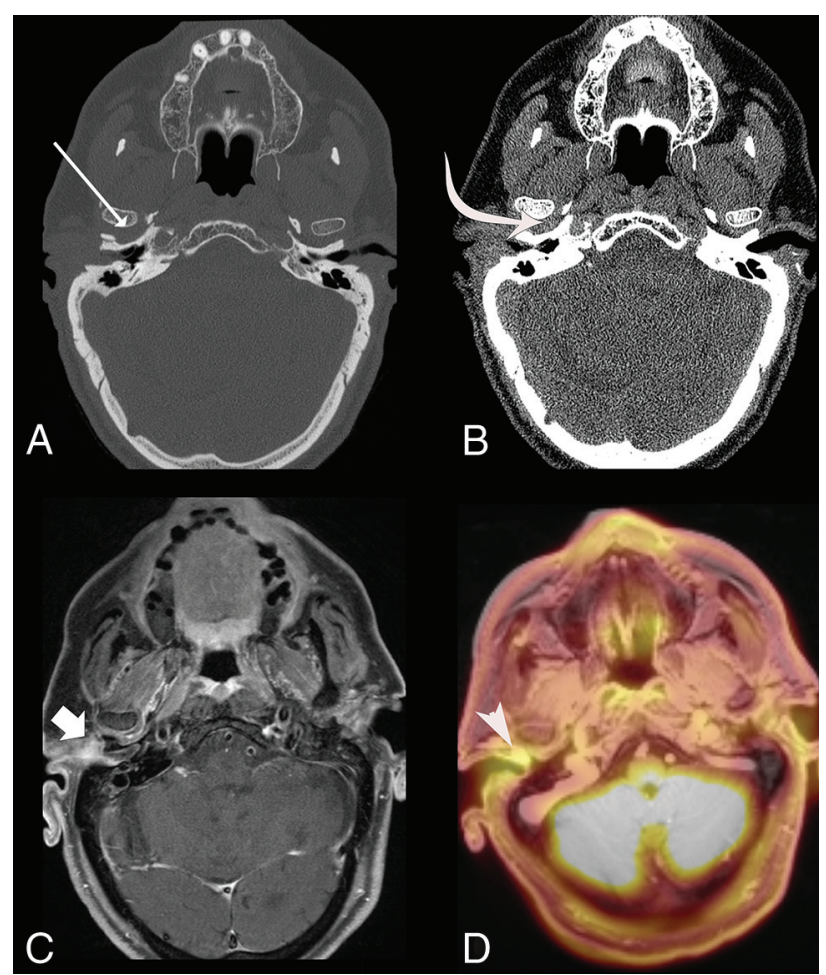

FIG 1. A 79-year-old man with type II diabetes presented with a painful right ear and pain during mastication. CT shows an increased distance of the TMJ (A, white arrow) and infiltration of the retrocondylar fat pad and surrounding fat planes ( $B$, curved arrow). The contrastenhanced T1-weighted MR imaging shows enhancement of the retrocondylar fat pad (C, broad arrow), with increased FDG accumulation on PET/MR imaging ( $D$, arrowhead). The biopsy of the external ear canal was positive for $P$ Aeruginosa.

retrocondylar fat pad, which is part of the NEO anterior spreading pattern. ${ }^{6}$ Some patients with NEO have a variant anatomic structure called a persistent foramen of Huschke (foramen tympanicum), a dehiscence located antero-inferior to the osseous external auditory canal (EAC) and posterior-medial to the temporomandibular joint (Fig 3). The foramen of Huschke has been described in relation to EAC salivary fistulas, TMJ herniation, the facilitation of tumor spread, and drainage of odontogenic abscesses in the EAC. ${ }^{7-10}$

The infectious spread from the TMJ to the EAC via the foramen of Huschke may also occur the other way around. A persistent foramen of Huschke can also pose an alternative route for NEO, which will put the TMJ, masticator space, and parotid gland at risk. Identifying this anatomic variation in patients with NEO may help recognize NEO and its extension in early stages of disease, thus preventing consecutive spread to its surrounding tissues, aiding a positive outcome in morbidity and mortality. The goal of this study was to assess the prevalence and size of a persistent foramen of Huschke in patients with NEO and thereby determine its value for routine assessment.

\section{MATERIALS AND METHODS}

A PACS survey was conducted with the key words "skull base osteomyelitis," "necrotizing external otitis," and "malignant external otitis.” In total, 39 patients diagnosed with NEO on temporal bone high-resolution CT scans in 2005-2018 were retrospec-

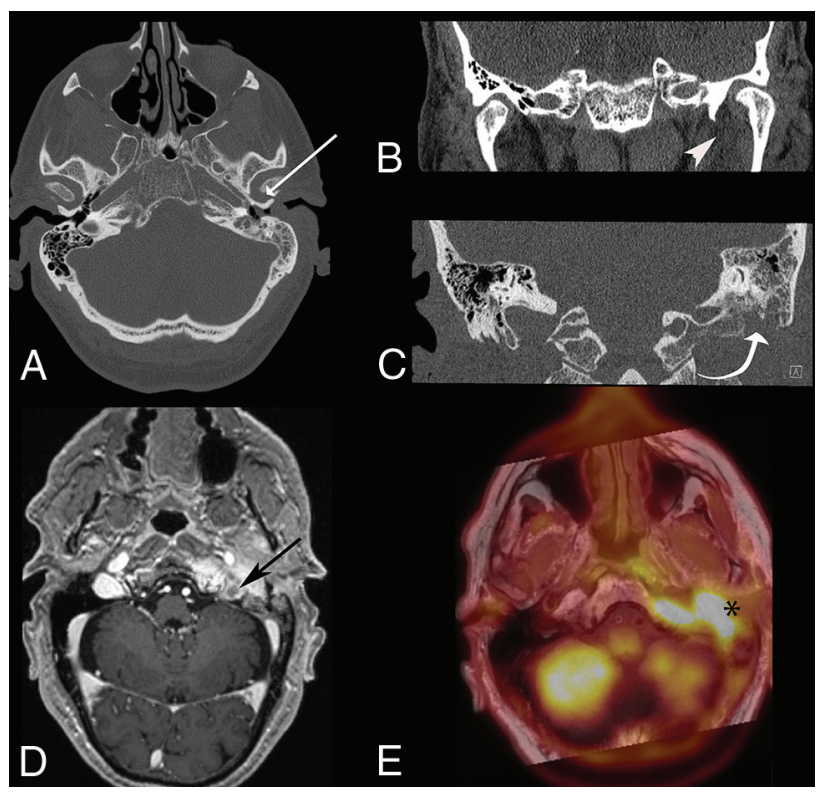

FIG 2. A 76-year-old man without a medical history presented with a painful ear and otorrhea. CT shows an increased distance of the TMJ ( $A$, white arrow), obliteration of subtemporal fat lines, and a retrocondylar fat pad sign (B. arrowhead), as well as osseous destruction of the mastoid ( $C$, white curved arrow). Contrast-enhanced T1-weighted image 2 weeks after diagnosis demonstrates progression of NEO with an anterior, medial, and intracranial spreading pattern complicated by a thrombus in the left jugular vein ( $D$, black arrow) with increased FDG accumulation on PET/MR imaging ( $E$, asterisk). History revealed noncompliance with antibiotic treatment. Biopsy of the external ear canal was positive for $P$ Aeruginosa.

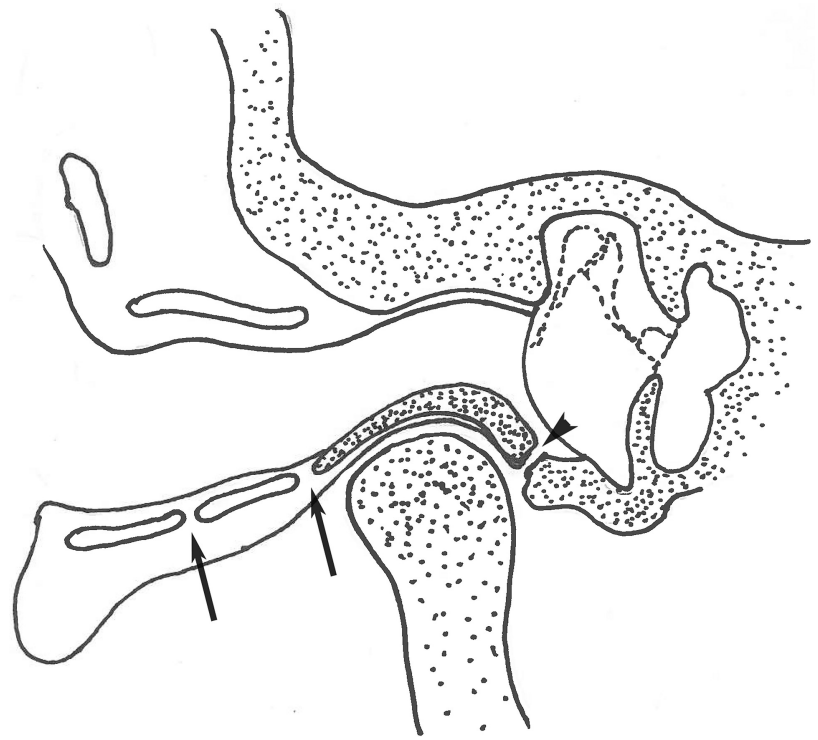

FIG 3. Coronal illustration of the location of the fissures of Santorini and the foramen of Huschke. The fissures of Santorini extend through the osteocartilaginous or cartilaginous junctions of the EAC (arrows). The foramen of Huschke (arrowhead) is located anterior-inferior to the EAC and posterior-medial to the temporomandibular joint.

tively included. All cases of NEO were confirmed by the Ear, Nose, and Throat Department in combination with EAC biopsy results positive for microbiological organisms associated with NEO. Most patients underwent MR imaging, which confirmed the diagnosis of NEO. Scans were assessed for the type of spreading 

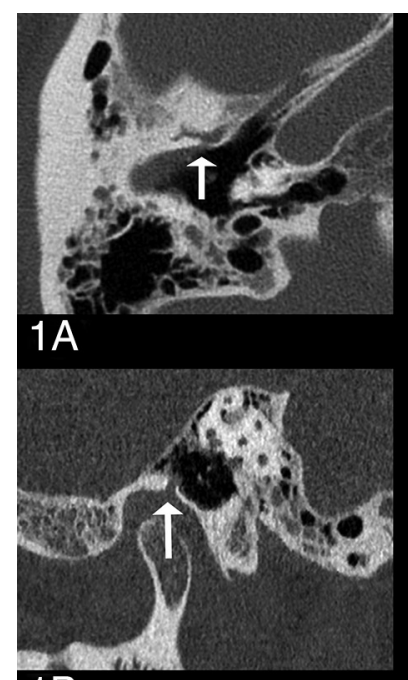

$1 \mathrm{~B}$

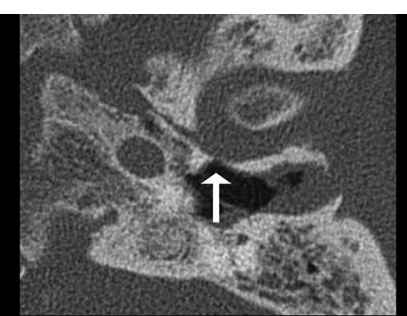

2A

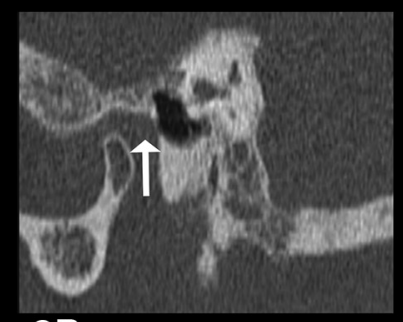

2B

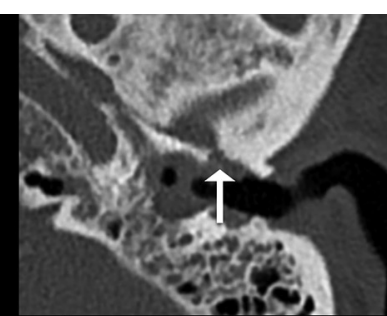

3A

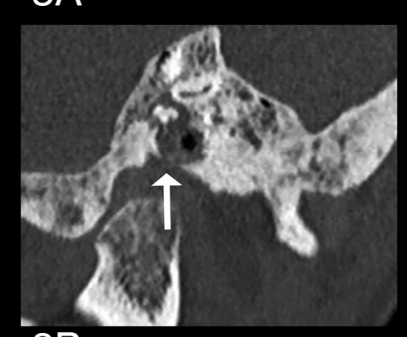

3B

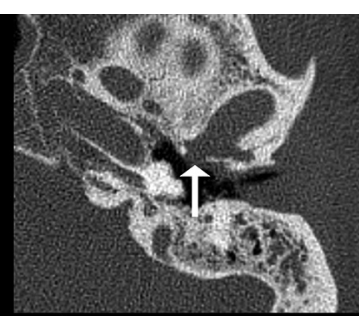

$4 \mathrm{~A}$

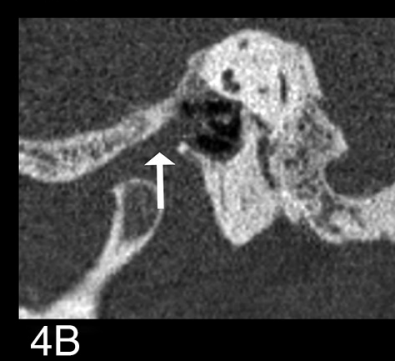

FIG 4. Temporal bone axial and sagittal views of patients with NEO with a persistent foramen of Huschke. The views show a subtle ( $7 A$ and $7 B)$, mild ( $2 A$ and $2 B)$, moderate $(3 A$ and $3 B)$, and extensive dehiscence ( $4 A$ and $4 B$ ) as indicated by the white arrows.

Table 1: Demographics for patients with NEO and controls ${ }^{a}$

\begin{tabular}{lccccc}
\hline & \multicolumn{2}{c}{ NEO $(\boldsymbol{n}=39)$} & & \multicolumn{2}{c}{$\begin{array}{c}\text { Controls } \\
(\boldsymbol{n}=39)\end{array}$} \\
\cline { 2 - 3 } \cline { 5 - 6 } $\begin{array}{c}\text { Age range } \\
\text { (Mean) }(\mathbf{y r})\end{array}$ & $\begin{array}{c}\text { Women } \\
(\boldsymbol{n}=11)\end{array}$ & $\begin{array}{c}\text { Men } \\
(\boldsymbol{n}=28)\end{array}$ & & $\begin{array}{c}\text { Women } \\
(\boldsymbol{n}=11)\end{array}$ & $\begin{array}{c}\text { Men } \\
(\boldsymbol{n}=28)\end{array}$ \\
\hline $50-59$ & $1(51)$ & $2(50)$ & & $1(59)$ & $2(56)$ \\
$60-69$ & $2(63)$ & $9(66)$ & & $2(63)$ & $9(65)$ \\
$70-79$ & $3(78)$ & $6(78)$ & & $3(74)$ & $6(74)$ \\
80 or older & $5(87)$ & $11(86)$ & & $5(84)$ & $11(84)$ \\
\hline
\end{tabular}

${ }^{a}$ Data are number of patients per age range.

pattern and the existence of a persistent foramen of Huschke on the first available CT of the temporal bone during the disease process. CT scans of the temporal bone in the control group with non-NEO or noninflammatory pathology (eg, hearing loss) were matched for age and sex. Control scans with former destruction of the skull (eg, severe cholesteatoma, radiation therapy, or severe otitis media) were excluded. NEO spreading patterns and the presence and/or absence of a foramen of Huschke were scored. The size of the persistent foramen of Huschke was measured as the maximum width in the axial plane and classified as subtle $(<0.5$ $\mathrm{mm})$, mild $(0.5-1.0 \mathrm{~mm})$, moderate $(1.0-1.5 \mathrm{~mm})$, or extensive $(>1.5 \mathrm{~mm}$ ) dehiscence (Fig 4). Interpretation and scoring were performed by a radiologist-in-training and a senior head and neck radiologist with $>10$ years' experience. Scoring discrepancies were solved via consensus. Statistical analyses were performed with SPSS, Version 25 (IBM, Armonk, New York). Frequency and Pearson $\chi^{2}$ tests were performed with significance levels set at $P<.05$.

\section{RESULTS}

\section{Foramen of Huschke}

Seventy-eight temporal bone CT studies were performed on 56 male and 22 female subjects with an overall mean age of $74.9 \pm$ 10.8 years (Table 1). A persistent foramen of Huschke was present in $28 / 78$ patients $(36 \%), 21 / 39$ patients with NEO and $7 / 39$ control subjects $\left(\chi^{2}=P<.05\right)$. A persistent foramen of Huschke occurred most often unilaterally and was bilateral in 5 of the 21
Table 2: Prevalence of the foramen of Huschke in patients with NEO and controls

\begin{tabular}{|c|c|c|c|c|c|}
\hline \multirow{2}{*}{$\begin{array}{c}\text { Foramen of } \\
\text { Huschke }\end{array}$} & \multicolumn{2}{|c|}{$\begin{array}{c}\text { NEO } \\
(n=39)\end{array}$} & \multicolumn{2}{|c|}{$\begin{array}{c}\text { Controls } \\
(n=39)\end{array}$} & \multirow[b]{2}{*}{$P<.05$} \\
\hline & No. & Percentage & No. & Percentage & \\
\hline Absent & 18 & $46 \%$ & 32 & $82 \%$ & $.002^{a}$ \\
\hline Present & 21 & $54 \%$ & 7 & $18 \%$ & $.002^{\mathrm{a}}$ \\
\hline Unilateral & 16 & $41 \%$ & 5 & $13 \%$ & - \\
\hline Bilateral & 5 & $13 \%$ & 2 & $5 \%$ & - \\
\hline Total & 39 & $100 \%$ & 39 & $100 \%$ & \\
\hline
\end{tabular}

Note:- - indicates data is not available.

a Significant.

Table 3: Location of the foramen of Huschke in patients with NEO and controls

\begin{tabular}{|c|c|c|c|c|}
\hline \multirow[b]{3}{*}{ Location } & \multicolumn{4}{|c|}{ Foramen of Huschke } \\
\hline & \multicolumn{2}{|c|}{ Present } & \multicolumn{2}{|c|}{ Absent } \\
\hline & No. & Percentage & No. & Percentage \\
\hline \multicolumn{5}{|l|}{ NEO } \\
\hline Left ear & 16 & $41 \%$ & 23 & $59 \%$ \\
\hline Right ear & 10 & $26 \%$ & 29 & $74 \%$ \\
\hline \multicolumn{5}{|l|}{ Controls } \\
\hline Left ear & 3 & $8 \%$ & 36 & $92 \%$ \\
\hline Right ear & 6 & $15 \%$ & 33 & $85 \%$ \\
\hline
\end{tabular}

patients with NEO and 2 of the control subjects, approximately a bilateral occurrence of $25 \%$ in both groups (Table 2). In our cohort, no preference for a left or right ear was found (Table 3 ).

The size of the foramen of Huschke in patients with NEO varied from subtle to extensive. The size was subtle in $6 / 26$, mild in $2 / 26$, moderate in $7 / 26$, and extensive in $11 / 26$ patients. In the latter, 10/11 extensive cases were seen in the affected NEO side (Table 4).

A persistent foramen of Huschke was present in 9 ears (11.5\%) of control subjects, of which 2 were bilateral (Table 2 ). The width of the foramen could be classified as subtle in 6/9, mild in $1 / 9$, and extensive in $2 / 9$ (Table 4 ). The mean foramen size of the patients with $\mathrm{NEO}$ was $2.9 \mathrm{~mm}$ in the axial plane (range, $0.2-14.9 \mathrm{~mm}$ ) versus $1.0 \mathrm{~mm}$ (range, $0.2-3.3 \mathrm{~mm}$ ) in control subjects. 
Table 4: Size of the persistent foramen of Huschke specified in controls and patients with NEO

\begin{tabular}{lcccc} 
& \multicolumn{3}{c}{ NEO } & \\
\cline { 2 - 4 } & $\begin{array}{c}\text { Affected } \\
\text { Nonaffected } \\
\text { Side/Ears } \\
(\boldsymbol{n}=\mathbf{3 8})\end{array}$ & $\begin{array}{c}\text { NEO } \\
\text { Ears } \\
(\boldsymbol{n}=\mathbf{4 0})\end{array}$ & $\begin{array}{c}\text { Total } \\
\text { Fors } \\
(\boldsymbol{n}=\mathbf{n}=\mathbf{7 8})\end{array}$ & $\begin{array}{c}\text { Total } \\
\text { Control } \\
\text { Ears } \\
(\boldsymbol{n}=\mathbf{7 8})\end{array}$ \\
\hline Subtle $(<0.5 \mathrm{~mm})$ & 4 & 2 & 6 & 6 \\
Mild $(0.5-1.0 \mathrm{~mm})$ & 0 & 2 & 2 & 1 \\
Moderate $(1.0-1.5 \mathrm{~mm})$ & 1 & 6 & 7 & 0 \\
Extensive $(>1.5 \mathrm{~mm})$ & 1 & 10 & 11 & 2 \\
Total & 6 & 20 & 26 & 9 \\
\hline
\end{tabular}

\section{NEO}

Bilateral NEO was present in 1/39; and unilateral NEO, in 38/39 (97\%) patients, with a total of 40 affected ears. The anterior spreading pattern was present in all except 1 patient. Twelve patients with NEO showed an anterior spreading pattern in combination with a posterior, medial, or intracranial pattern. A persistent foramen of Huschke was present in 26 ears of patients with NEO, 5 of which showed bilateral foramina (Table 2); 50\% (20/ 40) of NEO-affected ears showed a persistent foramen of Huschke in comparison with $15.8 \%$ (6/38) of the unaffected ears (Table 4).

\section{DISCUSSION}

The tympanic membrane develops from a tympanic ring with 4 different ossification centers that fuse with the temporal bone. ${ }^{11,12}$ The process of ossification starts at 9 weeks in utero with fusion of the tympanic ring taking up to 5 years. ${ }^{11,12}$ An incomplete medial fusion of the tympanic ring causes a dehiscence located antero-inferior to the osseous external ear canal and posterior-medial to the temporomandibular joint, an anatomic variation also known as a persistent foramen of Huschke.

Several symptoms have been described in relation to a persistent foramen of Huschke. Patients can describe clear discharge often in relation to mastication caused by synovial fluid transport through a fistula between the EAC and the TMJ space. ${ }^{78}$ A more prominent dehiscence can cause ear pain or a clicking sensation during mastication because the TMJ retrodiscal or discal tissue protrudes within the EAC. ${ }^{13-15}$ The protrusion of soft tissue into the EAC through the foramen of Huschke can mimic a mass lesion in the EAC. The difference between TMJ soft-tissue herniation and mass lesions can be made with EAC inspection with the patient with an opened mouth because protruded TMJ tissue will retract in this position. ${ }^{13,14}$ As previously mentioned, the foramen of Huschke has been described in relation to infectious or tumorous spread from the TMJ to the EAC. ${ }^{9,10}$ The expansion of the external otitis through the foramen of Huschke to the TMJ was first suggested in 1992, showing discharge in the TMJ without osseous destruction, which can be regarded as one of the earliest stages in NEO. ${ }^{16}$

The classic theory of necrotizing external otitis concerns the fissures of Santorini. External otitis can progress via the fissures of Santorini or a temporal bone dehiscence to developing NEO. The relation of the fissures of Santorini as an anterior spreading route for NEO is often suggested, but the fissures are not visible on current imaging techniques due to the fissures of Santorini location and subtle nature. Fistula discharge attributed to the fissures of Santorini often shows positive biochemical results for amylase,

indicating a connection with the parotid gland. ${ }^{7}$ In the case of NEO, one would expect soft-tissue changes of the parotid gland to precede TMJ changes because the osteocartilaginous junction lies more lateral in the EAC. However, of the 38 patients included in our study with an anterior spreading pattern, 11 showed involvement of the parotid space and 34 showed involvement of the TMJ, favoring an infectious spreading via the persistent foramen of Huschke instead or in association with the fissures of Santorini. Thus, an anatomic variation can also be an additional risk factor for progression to NEO.

The prevalence of a persistent foramen of Huschke in the literature ranges from $4.6 \%$ to $20 \%$, with a higher prevalence for women and the left ear. ${ }^{17-20}$ The prevalence in the control subjects and unaffected ears in our study is in line with these numbers; however, we did not find a left-sided predominance. We did not investigate a sex difference because the control subjects were sexmatched with the patient group. The prevalence of a persistent foramen in our patient group was $50 \%$ in NEO-affected ears; therefore, the prevalence in the diseased ears is far higher than in the healthy population.

Our results support the theory that the foramen of Huschke is a contributing risk factor in the development of necrotizing external otitis because the prevalence of $50 \%$ in patients with NEO lies well above the described incidence of $4.6 \%-38 \%$ in overall imaging and cadaver studies. ${ }^{11,12,21}$ The prevalence of a persistent foramen of Huschke has not been investigated in patients with NEO before, to our knowledge. Yeheskeli et $\mathrm{al}^{22}$ investigated the role of involvement of the TMJ in necrotizing external otitis and found that involvement of the TMJ served as a positive clinical factor. The prevalence of TMJ involvement in their study was comparable with the prevalence of the foramen of Huschke; however, the authors did not study the foramen prevalence. ${ }^{22}$ Our results show that patients with NEO, in addition to a foramen prevalence of $50 \%$ at the affected side, often show an extensive dehiscence $(>1.5 \mathrm{~mm})$ in contrast to the control group and unaffected ears.

It is difficult to discern whether the dehiscence in patients with NEO is the result of incomplete fusion of the tympanic ring or due to (primary or secondary) bone erosion from the underlying infectious process. Possible erosive changes can therefore skew the trend to a more severe classification. However, an extensive dehiscence was a finding not only in patients with NEO, it was also present in 2/9 control patients. True differentiation between primary bone erosion or incomplete fusion for patients with NEO can only be realized when comparing a respective patient's NEO scan with a preexistant non-NEO afflicted temporal bone scan. The reviewed CT scans were often the first imaging study of the temporal bone. Therefore, the difference between incomplete fusion or bone erosion could not be further distinguished.

One could regard the sample size as a limitation in this study; however, past studies dealt with a corresponding sample size due to the rare nature of NEO. This study with 39 cases of NEO lies well above the average case number per NEO series (22 cases) as reported by a NEO systematic review study. ${ }^{23}$ Due to the rare nature of the disease, a prospective study was not feasible to perform in a single center; therefore, we opted for a retrospective cohort design. 
The foramen of Huschke has a specified location but not a specified size; therefore, the region should be intensively studied so as not to miss subtle foramina. Cadaver studies showed a variation of the foramen width in the range of $1-5 \mathrm{~mm} .{ }^{11}$ High-resolution CT with a voxel size of $0.25 \mathrm{~mm}^{3}$ can even detect smaller foramina ranging from $0.2 \mathrm{~mm} .{ }^{19}$ The included CT petrosal scans in our studies were obtained between 2005 and 2018, during which the CT technique, among other parameters, showed a great increase in spatial resolution. The slice thickness used in our study is heterogeneous, ranging from 0.4 to $1 \mathrm{~mm}$, thus leading to a possible underestimation of subtle foramina of Huschke in some patients with NEO. This was not a problem in the current study because the prevalence of the foramen of Huschke in the control group and unaffected ears is in line with literature. Prevalence in the affected NEO ears could potentially be higher.

We demonstrated in our study that the prevalence of a persistent foramen of Huschke is higher in patients with skull base involvement in necrotizing external otitis compared with the healthy population, suggesting a role in the pathophysiology of NEO. Identification of a persistent foramen of Huschke at imaging can help in early identification of an anterior spreading pattern in NEO.

\section{CONCLUSIONS}

An anterior spreading pattern from a necrotizing external otitis is associated with the presence and increased size of a persistent foramen of Huschke. These findings suggest a role for the persistent foramen of Huschke next to the fissures of Santorini. Careful inspection in necrotizing external otitis in the region of the foramen of Huschke could identify anterior spread to the skull base in an early stage, aiding in improved patient care and outcome.

Disclosures: W.L. van der Meer-UNRELATED: Employment: Radiology Resident at Maastricht Medical University Center since November 2016. Mark van TilburgUNRELATED: Employment: Ear, Nose, and Throat resident.

\section{REFERENCES}

1. Rubin Grandis J, Branstetter BF 4th, Yu VL. The changing face of malignant (necrotising) external otitis: clinical, radiological, and anatomic correlations. Lancet Infect Dis 2004;4:34-39 CrossRef Medline

2. Soudry E, Hamzany Y, Preis M, et al. Malignant external otitis: analysis of severe cases. Otolaryngol Head Neck Surg 2011;144:758-62 CrossRef Medline

3. Glikson E, Sagiv D, Wolf M, et al. Necrotizing otitis externa: diagnosis, treatment, and outcome in a case series. Diagn Microbiol Infect Dis 2017;87:74-78 CrossRef Medline

4. Kwon B, Han M, Oh S, et al. MRI findings and spreading patterns of necrotizing external otitis: is a poor outcome predictable? Clin $\mathrm{Ra}$ diol 2006;61:495-504 CrossRef Medline

5. van Kroonenburgh A, van der Meer W, Bothof R, et al. Advanced imaging techniques in skull base osteomyelitis due to malignant otitis externa. Curr Radiol Rep 2018;6:1-14 CrossRef Medline

6. Lee J, Song, J, Oh S, et al. Prognostic value of extension patterns on follow-up magnetic resonance imaging in patients with necrotizing otitis externa. Arch Otolaryngol Head Neck Surg 2011;137:688-93 CrossRef Medline

7. Rana K, Rathore P, Raj A, et al. Bilateral spontaneous salivary otorrhoea: case report and a review of the literature. Int $\mathrm{J}$ Pediatr Otorhinolaryngol 2015;79:1774-77 CrossRef Medline

8. Ajduk J, Ries M, Vagic D, et al. Temporomandibular joint fistula into the external ear canal. J Laryngol Otol 2012;126:837-39 CrossRef Medline

9. Jaber S, Rudic M, Keogh I. Pleomorphic adenoma of the external auditory canal: a rare presentation. Case Rep Otolaryngol 2015;2015: 696531 CrossRef Medline

10. Pepato A, Yamaji M, Sverzut C, et al. Lower third molar infection with purulent discharge through the external auditory meatus: case report and review of literature. Int J Oral Maxillofac Surg 2012;41: 380-83 CrossRef Medline

11. Bhanu PS, Sankar KD. Incidence of foramen of Huschke in South Andhra population of India. J Clin Diagn Res 2016;10:1-3 CrossRef Medline

12. Lacout A, Marsot-Dupuch K, Smoker W, et al. Foramen tympanicum, or foramen of Huschke: pathologic cases and anatomic CT study. AJNR Am J Neuroradiol 2005;26:1317-23 Medline

13. Kim T, Lee S, Kim S, et al. A case of spontaneous temporomandibular joint herniation into the external auditory canal with clicking sound. Korean J Audiol 2013;17:90-93 CrossRef Medline

14. Burlak K, So T, Maclaurin W, et al. Foramen tympanicum with symptomatic temporomandibular joint herniation. Radiol Case Rep 2018;13:822-24 CrossRef Medline

15. Heffez L, Anderson D, Mafee M. Developmental defects of the tympanic plate: case reports and review of the literature. J Oral Maxillofac Surg 1989;47:1336-40 CrossRef Medline

16. Dingle A. Fistula between the external auditory canal and the temporomandibular joint: a rare complication of otitis externa. $J$ Laryngol Otol 1992;106:994-95 CrossRef Medline

17. Tozoğlu U, Caglayan F, Harorlı A. Foramen tympanicum or foramen of Huschke: anatomical cone beam CT study. Dentomaxillofac Radiol 2012;41:294-97 CrossRef Medline

18. Pẹkala J, Pẹkala P, Satapathy B, et al. Incidence of foramen Tympanicum (of Huschke): comparing cadaveric and radiologic studies. $J$ Craniofac Surg 2018;29:2348-52 CrossRef Medline

19. Deniz Y, Geduk G, Zengin A. Examination of foramen tympanicum: an anatomical study using cone-beam computed tomography. Folia Morphol (Warsz) 2018;77:335-39 CrossRef Medline

20. Hashimoto T, Ojiri H, Kawai Y. The foramen of Huschke: age and gender specific features after childhood. Int J Oral Maxillofac Surg 2011;40:743-46 CrossRef Medline

21. Akbulut N, Kursun S, Aksoy S, et al. Evaluation of foramen Tympanicum using cone-beam computed tomography in orthodontic malocclusions. J Craniofac Surg 2014;25:105-09 CrossRef Medline

22. Yeheskeli E, Eta R, Gavriel H, et al. Temporomandibular joint involvement as a positive clinical prognostic factor in necrotising external otitis. J Laryngol Otol 2016;130:435-39 CrossRef Medline

23. Mahdyoun P, Pulcini C, Gahide I, et al. Necrotizing otitis externa: a systematic review. Otol Neurotol 2013;34:620-29 CrossRef Medline 